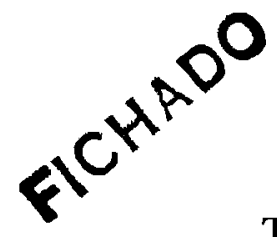

BIBL. JORGE WILLIAMS

$068<\quad 133$

TAXONOMIC AND EVOLUTIONARY SIGNIFICANCE OF BIOGENIC AMINES AND POLYPEPTIDES OCCURRING IN AMPHIBIAN SKIN. I. NEOTROPICAL

LEPTODACTYLID FROGS

J. M. Cei, V. Erspamer and M. Roseghini

Reprinted from Systematic Zoolocy

Vol. 16, No. 4, December 18, 1967, pp. 328-342

Made in United States of America 


\title{
TAXONOMIC AND EVOLUTIONARY SIGNIFICANCE OF BIOGENIC AMINES AND POLYPEPTIDES OCCURRING IN AMPHIBIAN SKIN. I. NEOTROPICAL LEPTODACTYLID FROGS
}

\author{
J. M. Cei, V. Erspanier and M. Roseghini
}

\begin{abstract}
Qualitative identification and quantitative estimation both of aromatic biogenic amines and pharmacologically active polypeptides in the skin of amphibians appear to offer a valuable contribution to studies in taxonomy and evolution of this vertebrate class.

Several examples of the accordance of the biochemical data herein reported with the data of traditional taxonomy are described for the neotropical family of frogs Leptodactylidae, but particular emphasis is laid on the striking correspondence between the systemic distribution of the spectra of biogenic amines and morphological taxonomy which is demonstrated by the species of the genus Leptodactylus.
\end{abstract}

During the past eight years paper chromatographic and biological screening of approximately 300 amphibian species was carried out. Specimens were collected throughout the world, but especially in South and North America.

The tissue subjected to screening was the skin, from which methanol or acetone extracts were prepared. Skin and its secretory activity are of tremendous importance in amphibian biology. In fact, the integumentary wall possesses a number of protective and adaptative, ecological, and behavioral functions, displays essential respiratory activity, and is the site of much passage of water and electrolytes.

Compounds which attracted our particular attention were aromatic biogenic amines (indolealkylamines, imidazolealkylamines, and phenylalkylamines) on the one side, and polypeptides active on plain muscle and/or external secretions on the other side.

Our results were beyond any expectation. In fact, amphibian skin was found to contain, on the whole, a formidable array of amines and polypeptides. Not only was the widespread occurrence and distribution of known amines confirmed, but several new amines and polypeptides were identified, often with their metabolites.

Analytical data on our pharmacological and biochemical studies have been pub- lished and will be published elsewhere (Erspamer, Cei, and Roseghini, 1963; 1964; Erspamer, Vitali, Roseghini, and Cei, 1963; 1964; Bertaccini, Cei, and Erspamer, 1965a; 1965b; Anastasi, Erspamer, and Bertaccini, 1965; Erspamer, Bertaccini, and Urakawa, 1964; Erspamer, Anastasi, Endean, and Roseghini, 1966; Erspamer, De Caro, and Endean, 1966).

In the present series of papers we wish to utilize the results of our research to elucidate the biochemical taxonomy of amphibians.

In fact, the occurrence of a given amine, a given amine metabolite, or a given amine precursor requires the activity of the enzyme system(s) responsible for their biosynthesis. Very often these enzymes are highly specific. But enzymes are proteins, i.e., precise chemical structures built from regular subunits under the control of the genetic code. For polypeptides we can perhaps go even further, because every single sequence of amino acids not only is genetically determined, but also can give us some information about the gene which directed its synthesis.

It is obvious that each amine, and each amino acid link, is a trait by which we can trace species evolution. Thus, biochemical taxonomy, in its broadest sense, has possibly even a greater validity than traditional 
taxonomy, based on somatic or osteological structures, size or coloration ( $\mathrm{Cei}$ and Erspamer, 1966).

In the present paper we will report and discuss a number of biochemical and pharmacological data concerning the skin of leptodactylid frogs. Successive papers will deal with other amphibian families.

The superfamily Leptodactylidae (Berg, 1896) comprises a great number of procoelid frogs, but it lacks real homogeneity in spite of some shared characteristics. This is in agreement with the frequently diverse taxonomic criteria used to determine the position of its subfamilies (cf. Gallardo, 1965) and the uncertain validity of many of its genera. Noble (1931) considered these frogs to be a neotropical branch of the Bufonidae, but the taxonomic of the family Leptodactylidae has been unquestionably accepted in recent treatises, such as the work of Goin and Goin (1962), in which the austral affinities of this group are pointed out. Goin and Goin reunited in a subfamily (Leptodactylinae) most of the South American genera. The neotropical genera Sminthillus, Geobatrachus, Euparkella, Rhinoderma, and Noblella belong to the Rhinodermatinae, the Brazilian Elosia, Megaelosia, and Crossodactylus to the Elosinae, the scarce African forms of Heleophrynus to the Heleophryninae, and to the Cycloraninae and Myobatrachynae the isolated but highly differentiated Australian "criniid" frogs.

Subfamilies formerly proposed or discussed for the leptodactylid branch were also the Telmatobiinae (Vellard, 1951; Burger, 1954), the Calyptocephalellinae (Reig, 1959), and the Eleutherodactylinae. In agreement with Lutz (1954) a subfamily Cyclorhamphinae (Cyclorhamphus, Thoropa) and with Gallardo (1965) a subfamily Batrachylinae (Eupsophus, Batrachyla) probably could be considered. The Pseudinae of Noble (1931) is no longer recognized, but its few genera (Pseudis, Lysapsus) now are placed in the family Pseudidae (Savage and Carvalho, 1953) closely related to the hylid frogs. The validity of Ceratoph- rydae (Miranda Ribeiro, 1926) may be supported, and because of its general leptodactylid affinity this group will be reported in our discussion. Detailed studies on Australian "criniid" frogs will be presented elsewhere.

\section{MATERIALS AND METHODS}

The species considered in this paper, together with data concerning number and state of the skins, solvent used for extraction, date and place of collection are indicated in the tables.

Fresh and/or dry skins were studied. Extracts of fresh material were prepared by extracting twice with 5 parts $(\mathrm{w} / \mathrm{v})$ of methanol the skins removed from the animals immediately after their sacrifice. The skins were spread out and dried in the shade as rapidly as possible. Immediately after their arrival in Italy, by air mail, they were minced with scissors and then immersed in 15-20 parts of the solvent. The extracts were combined and filtered. When kept in dark bottles and refrigerated, they may be stored for months and even years without appreciable loss of amines or polypeptide activity.

Methanol and acetone extracts were subjected to paper chromatographic and biological screening either as crude extracts or after having been partially purified by passage through an alumina column. Of course, particularly interesting materials were subjected to thorough investigation for the isolation of the active constituents and the elucidation of their chemical structure.

Descriptions of our paper chromatographic and biological procedures are given in other papers (Erspamer and Falconieri Erspamer, 1962; Erspamer and Glässer, 1963; Erspamer, Roseghini, and Cei, 1964; 1967; Erspamer, Vitali, Roseghini, and Cei, $1964 ; 1967)$.

Here we wish only to point out that, whereas bioassay methods may be considered really quantitative, paper chromatographic methods are only of semiquantitative value. In fact, estimations of the intensities and sizes of the individual col- 
Table 1. The CONTENT OF BIOgenic amines (IN $\mu$ g PER g DRY TISSUE) AND POLYPEPTIDES IN THE SKIN OF LEPTODACTYLIDS.

\begin{tabular}{|c|c|c|c|c|}
\hline Taxa, sample size, locality, date & $\begin{array}{c}\begin{array}{c}\text { Indole- } \\
\text { alkylamines } \\
(5 \mathrm{HT})\end{array} \\
\end{array}$ & $\begin{array}{l}\text { Phenyl-alkyl- } \\
\text { amines (lep- } \\
\text { todactyline) }\end{array}$ & $\begin{array}{l}\text { Imidazole- } \\
\text { alkylamine }\end{array}$ & Polypeptides \\
\hline \multicolumn{5}{|l|}{ Telmatobiinae } \\
\hline $\begin{array}{l}\text { Batrachophrynus macrostomum } \\
\text { (6) Perú, Junín Lake, } 4200 \mathrm{mtr} .1963,1(\text { MET })^{1}\end{array}$ & - & - & - & - \\
\hline $\begin{array}{l}\text { Batrachophrynus patagonicus } \\
\text { (8) Argentina, Neuquén, Laguna Blanca, } 1270 \\
\text { mtr. 1963, } 1 \text { (MET) }\end{array}$ & - & - & - & - \\
\hline $\begin{array}{l}\text { Telmatobius montanus } \\
\text { (3) Argentina, Mendoza, Pehuenche Pass, } 2500 \\
\text { mtr. 1964, } 3 \text { (AC) }\end{array}$ & - & $0.4-0.8$ & - & - \\
\hline $\begin{array}{l}\text { Telmatobius hauthali } \\
\text { (52) Argentina, Tucumán, Tafí Valley, } 2500 \\
\text { mtr. 1959, 12 (AC) }\end{array}$ & - & - & - & - \\
\hline $\begin{array}{l}\text { (14) Argentina, Tucumán, Tafí Valley, } 2500 \\
\text { mtr. 1961, } 12 \text { (F) (MET) }\end{array}$ & - & 0.4 & - & - \\
\hline $\begin{array}{l}\text { Telmatobius halli } \\
\text { (29) Chile, Antofagasta, S. Pedro, } 2500 \mathrm{mtr} . \\
1965,10 \text { (MET) }\end{array}$ & - & - & - & - \\
\hline $\begin{array}{l}\text { Telmatobius jelskii } \\
\text { (3) Perú, Arequipa, } 2250 \mathrm{mtr} .1963,2 \text { (MET) }\end{array}$ & - & - & 一 & $\begin{array}{l}\text { (presence of a } \\
\text { physalaemin-like } \\
\text { polypeptide) }\end{array}$ \\
\hline \multicolumn{5}{|l|}{ Calyptocephalellinae } \\
\hline $\begin{array}{l}\text { Calyptocephalella gayi } \\
\text { (5) Chile, Santiago, 1961, } 1 \text { (AC) }\end{array}$ & - & 5.3 & - & - \\
\hline \multicolumn{5}{|l|}{ Elosiinae } \\
\hline $\begin{array}{l}\text { Elosia aspera } \\
\text { (40) Brazil, S. Paulo, 1962, } 1 \text { (F) (MET) }\end{array}$ & 一 & 0.4 & - & - \\
\hline $\begin{array}{l}\text { Elosia lateristrigata } \\
\text { (11) Brazil, S. Paulo, 1962, } 1 \text { (F) (MET) }\end{array}$ & - & 0.4 & - & - \\
\hline \multicolumn{5}{|l|}{ Cyclorhamphinae } \\
\hline $\begin{array}{l}\text { Cyclorhamphus fuliginosus } \\
\text { (26) Brazil, S. Paulo, 1962, 1 (AC) }\end{array}$ & - & - & - & - \\
\hline $\begin{array}{l}\text { Thoropa miliaris } \\
\text { (26) Brazil, S. Paulo, 1962, I (AC) }\end{array}$ & $6-8$ & $0.8-1.5$ & - & - \\
\hline $\begin{array}{l}\text { Thoropa petropolitana } \\
\text { (75) Brazil, Rio Janeiro, 1962, } 4 \text { (MET) }\end{array}$ & ? & $<\mathrm{I}$ & - & - \\
\hline $\begin{array}{l}\text { Batrachylinae } \\
\text { Eupsophus roseus } \\
\text { (6) Argentina, Lacar Lake, 1964, I (MET) }\end{array}$ & - & 0.6 & - & - \\
\hline $\begin{array}{l}\text { Eupsophus nodosus } \\
\text { (3) Chile, Zapallar, 1963, } 12 \text { (MET) }\end{array}$ & - & 0.85 & - & - \\
\hline \multicolumn{5}{|l|}{ Eleutherodactylinae } \\
\hline $\begin{array}{l}\text { Eleutherodactylus ranoides } \\
\text { (8) Costa Rica, S. José, 1962, } 4 \text { (MET) }\end{array}$ & - & - & - & - \\
\hline $\begin{array}{l}\text { Eleutherodactylus bufoniformis } \\
\text { (1) Panamá, Barro Col. Isl., 1964, } 1 \text { (MET) }\end{array}$ & - & - & - & - \\
\hline
\end{tabular}

1 (F), fresh skin; (AC), acetone extraction; (MET), methanol extraction; -, not detected. 
ored spots produced on paper chromatograms by different amounts of crude or semi-purified skin extracts were made chiefly by visual comparison with spots produced by known amounts of the corresponding pure synthetic or natural compounds.

The problem of whether the dry skin does reproduce the true qualitative and quantitative spectrum of biogenic amines and polypeptides occurring in the fresh skin, i.e., in the living tissue, has been faced in appropriate experiments (Erspamer, Roseghini, and Cei, unpublished data; Erspamer, Roseghini, and Endean, unpublished data), from which the following conclusions may be drawn:

a) Drying of the skin does not change the spectrum of biogenic amines or active polypeptides, i.e., no compound of any importance is found in dried skin that is not found in fresh skin, and vice versa.

b) It is, however, possible that during drying there occurs occasionally a quantitative variation in the concentration of some constituents. This variation may be in the direction of a decrease (e.g., polypeptides, 5-HT) or more rarely, of an increase (again 5-HT, 5-HIAA, histamine). These changes, which apparently may affect only some of the amines and polypeptides, may be easily explained by the persisting activity of some skin enzymes during drying, especially in the first phases of the drying process.

The above observations are of decisive importance, for they confirm the full validity of all the qualitative data obtained in dried skins. Quantitative data are in part less reliable, but it is obvious that they also retain great comparative validity, especially if dried skins are compared with dried skins and fresh skins with fresh skins.

\section{RESULTS}

Results of paper chromatographic and biological screening are presented in tables 1 to 5 .

South-American subfamilies of leptodactylid frogs are arranged in these tables in accord with accepted systematic criteria, especially that of the recent treatise by Goin and Goin (1962).

The tables indicate, for each species, number of specimens examined (with a statement if screening has been carried out on single specimens or on pools), the solvent used for extraction (methanol or acetone), and finally locality and date (year and month) of collection.

Unless otherwise stated, dried skins were examined. Fresh skins were indicated with (F). Whereas acetone (AC) proved to be the best solvent for indolealkylamines, phenyl-alkylamines, and spinaceamines, methanol (MET) was the most suitable solvent for histamines and polypeptides. The appropriate choice of the solvent is particularly important for a quantitative extraction of histamines, spinaceamines, and polypeptides, but is less important for the other compounds.

\section{DISCUSSION} I

Biogenic aromatic amines and active peptides found in the skin of the leptodactylid frogs examined in the present study were as follows:

1. ( $\alpha$ ) indolealkylamines: 5-hydroxytryptamine (5-HT), N-methyl 5-HT, bufotenidine, dehydrobufotenine; $(\beta)$ imidazolealkylamines: histamine, $N$-acetylhistamine, $N$-methylhistamine, N.N-dimethylhistamine, spinaceamine, 6-methylspinaceamine; $(\gamma)$ phenylalkylamines: $p$-tyramine, leptodactyline ( $m$-hydroxyphenylethyltrimethylammonium).

2. physalaemin; caerulein-like peptides. The occurrence of the above amine spectrum demands the activity of the following enzyme systems: tryptophan 5-hydroxylase, the enzyme responsible for the introduction of the $-\mathrm{OH}$ group in the 5 position of the indole nucleus of tryptophan, i.e., responsible for the biosynthesis of 5-hydroxytryptophan, the obligatory precursor of all 5hydroxyindolealkylamines; decarboxylase $(s)$ of aromatic L-aminoacids, capable of decarboxylating 5-hydroxytryptophan to 5-HT, histidine to histamine, para- and meta- 


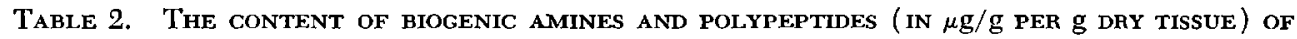
THE SKIN OF LEPTODACTYLIOS.

\begin{tabular}{|c|c|c|c|c|}
\hline Taxa, sample size, locality, date & $\begin{array}{c}\text { Indole- } \\
\text { alkylamines } \\
(5 \mathrm{HT})\end{array}$ & $\begin{array}{l}\text { Phenyl-alkyl- } \\
\text { amines (lep- } \\
\text { todactyline) }\end{array}$ & $\begin{array}{l}\text { Imidazole- } \\
\text { alkylamines }\end{array}$ & Polypeptides \\
\hline \multicolumn{5}{|l|}{ Erhinodermatinae } \\
\hline $\begin{array}{l}\text { Rhinoderma darwini } \\
\text { (35) Chile, Concepción, 1965, } 10(\mathrm{~F})(\mathrm{MET})^{1}\end{array}$ & 230 & - & - & - \\
\hline \multicolumn{5}{|l|}{ Leptodactylinae } \\
\hline $\begin{array}{l}\text { Physalaemus biligonigerus }{ }^{2} \\
\text { (745) Argentina, Tucumán, Summer 1959- } \\
1964 \text { (4 pools) (MET) }\end{array}$ & - & 2.5 & - & $370-700$ \\
\hline $\begin{array}{l}\text { (471) Argentina, Tucumán, 1963, } 1 \text { (F) } \\
\text { (MET) }\end{array}$ & - & scarce & - & 150 \\
\hline $\begin{array}{l}\text { (53) Argentina, Córdoba, 1963, } 12 \text { (F) } \\
\text { (MET) }\end{array}$ & - & scarce & - & 75 \\
\hline $\begin{array}{l}\text { Physalaemus centralis } \\
\text { (10) Brazil, Rondonia, 1962, } 11 \text { (MET) }\end{array}$ & - & - & - & $300-400$ \\
\hline $\begin{array}{l}\text { Physalaemus bresslaui } \\
\text { (97) Brazil, Río de Janeiro, 1963, } 12 \text { (MET) }\end{array}$ & - & - & - & $15-35$ \\
\hline $\begin{array}{l}\text { Physalaemus cuvieri } \\
\text { (2) Argentina, Misiones, 1961, } 2 \text { (MET) }\end{array}$ & - & - & - & 0.1 \\
\hline $\begin{array}{l}\text { Eupemphix nattereri } \\
\text { (9) Brazil, Matto Grosso, 1962, } 12 \text { (MET) }\end{array}$ & - & - & - & - \\
\hline $\begin{array}{l}\text { Pleurodema cinerea } \\
\text { (33) Argentina, Tucumán, Summer 1959-1961 } \\
\text { (4 pools) (AC) }\end{array}$ & - & $4.8-11$ & - & - \\
\hline $\begin{array}{l}\text { (52) Argentina, Tucumán, 1961, 12;1963, } 1 \\
\text { (2 pools) (F) (MET) }\end{array}$ & - & $0.9-2.2$ & - & - \\
\hline (5) Bolivia, La Paz, 3800 mtr. 1963, 1 (MET) & - & 0.6 & - & - \\
\hline $\begin{array}{l}\text { Pleurodema tucumana } \\
\text { (186) Argentina, Tucumán, Summer 1959- } \\
1961 \text { (4 pools) (AC) }\end{array}$ & - & $4-7$ & - & - \\
\hline $\begin{array}{l}\text { (74) Argentina, Tucumán, Summer } 1962 \text { (F) } \\
\text { (MET) }\end{array}$ & - & 1 & - & - \\
\hline $\begin{array}{l}\text { Pleurodema guayapae } \\
\text { (16) Argentina, La Rioja, Catuna, 1964, } 11 \\
\text { (MET) }\end{array}$ & - & 0.4 & - & - \\
\hline $\begin{array}{l}\text { (44) Argentina, Santiago del Estero, Sala- } \\
\text { dillo River, 1964, } 12 \text { (MET) }\end{array}$ & - & - & - & - \\
\hline $\begin{array}{l}\text { Pleurodema nebulosa } \\
\text { (1) Argentina, Mendoza, 1961, } 2 \text { (AC) }\end{array}$ & - & 2 & - & - \\
\hline $\begin{array}{l}\text { Pleurodema bibroni } \\
\text { (140) Chile, Santiago, 1960, } 7 \text { (AC) }\end{array}$ & 0.6 & 5.3 & - & - \\
\hline (15) Argentina, Lacar Lake, 1964, 1 (AC) & - & 5.3 & - & - \\
\hline $\begin{array}{l}\text { Pleurodema bufonina } \\
\text { (5)Argentina, Neuquén, Laguna Blanca Lake, } \\
1964,2 \text { (AC) }\end{array}$ & $240^{3}$ & - & - & - \\
\hline $\begin{array}{l}\text { (6) Argentina, Santa Cruz, Gregores, } 1966,3 \\
\text { (F) (MET) }\end{array}$ & $30(b), 3-4^{4}$ & - & - & - \\
\hline
\end{tabular}

1 (MET), methanol extraction; (AC), acetone extraction; (F), fresh skin; -, not detected. 2 Cited as fuscumaculatus in previous works.

3 Inguinal glands only.

4 Total skin minus inguinal glands. 
TABle 3. The CONTENT OF BIOGENIC AMINES AND POLYPEPTIDES (IN $\mu \mathrm{g}$ PER g DRY TISSUE) IN THE SKIN OF LEPTODACTYLIDS.

\begin{tabular}{|c|c|c|c|c|c|c|}
\hline \multirow[b]{2}{*}{ Taxa, sample size, locality, date } & \multirow[b]{2}{*}{$5 \mathrm{HT}$} & \multicolumn{2}{|c|}{ Indolealkylamines } & \multirow{2}{*}{$\begin{array}{l}\text { Phenylalkyl- } \\
\text { amines (lepto- } \\
\text { dactyline) }\end{array}$} & \multirow{2}{*}{$\begin{array}{l}\text { Imidazole } \\
\text { alkylamines }\end{array}$} & \multirow{2}{*}{$\begin{array}{l}\text { Polypep- } \\
\text { tides (caeru- } \\
\text { lein-like) }\end{array}$} \\
\hline & & $\begin{array}{c}\text { N-Methyl } \\
\text { 5HT }\end{array}$ & $\begin{array}{l}\text { Bufote- } \\
\text { nidine }\end{array}$ & & & \\
\hline
\end{tabular}

\section{Leptodactylinae}

Leptodactylus

"Cavicola" Section

L. bufonius

(10) Argentina, Formosa, 1960, 3

$(\mathrm{AC})^{\mathbf{1}}$

(11) Argentina, Tucumán, 1961, 12

(F) (MET)

(6) Argentina, Santiago del Estero, 1964,12 (AC)

L. prognathus

(30) Argentina, Tucumán, Summer 1959 ( AC)

(48) Argentina, Tucumán, 1961, 12

(F) (MET)

L. sibilatrix

(1) Argentina, Chaco, 1963, 12 (F) (MET)

L. gracilis

(1) Argentina, Santa Fé, 1963, 11 (MET)

(3) Argentina, Córdoba, 1964, 12 (MET)

L. mystacinus

(2) Argentina, San Luis, 1964, 12 (AC)

"Platymantis" Section

L. caliginosus podicipinus

(44) Argentina, Paraná borders:

Resistencia, Corrientes, 1962, 10

(MET)

$180-640^{2} \quad-\quad 15-20 \quad 2700-5300 \quad-$

L. caliginosus petersi

(5) Perú, Tingo María, 1963, 1

(AC)

$1-1.5 \quad-$

$3 \quad 750$

L. melanonotus

(35) México, Vera Cruz, 1963, 10

(AC)

$35 \quad 10$

25

265

L. rubido

(2) Perú, Tingo María, 1963, 1 (AC)

- 40-45

200

60

L. curtus

(3) Perú, Tumbez, 1965, I (AC)

$70-80$

15

1200

50

1 (F), fresh skins; (MET), methanol extraction; (AC), acetone extraction; -, not detected.

2 Range of means of many pools. 
TABle 4. THE CONTENT OF AROMATIC AMINES AND POLYPEPTIDES (IN $\mu$ g PER g DRY OR FRESH TISSUE) IN THE SKIN OF LEPTODACTYLIDS.

\begin{tabular}{|c|c|c|c|c|c|c|c|c|c|c|}
\hline \multirow[b]{3}{*}{$\begin{array}{l}\text { Taxa, sample size, locality, } \\
\text { date }\end{array}$} & \multirow{2}{*}{\multicolumn{2}{|c|}{$\begin{array}{c}\text { Indolealkyl- } \\
\text { amines }\end{array}$}} & \multirow{2}{*}{\multicolumn{2}{|c|}{$\begin{array}{l}\text { Phenylalkyl- } \\
\text { amines }\end{array}$}} & \multicolumn{5}{|c|}{ Imidazolealkylamines } & \multirow{3}{*}{$\begin{array}{c}\text { Poly- } \\
\text { peptides } \\
\text { (caeru- } \\
\text { lein- } \\
\text { like) }\end{array}$} \\
\hline & & & & & \multirow[b]{2}{*}{$\begin{array}{c}\text { Hista- } \\
\text { mine }\end{array}$} & \multirow{2}{*}{$\begin{array}{l}\mathrm{N}- \\
\text { Methyl } \\
\text { hista- } \\
\text { mine }\end{array}$} & \multirow{2}{*}{$\begin{array}{l}\text { N,N-Di- } \\
\text { methyl } \\
\text { hista- } \\
\text { mine }\end{array}$} & \multirow{2}{*}{$\begin{array}{c}\text { Spinace- } \\
\text { amine }\end{array}$} & \multirow{2}{*}{$\begin{array}{l}\text { 6- } \\
\text { Methyl- } \\
\text { spinace- } \\
\text { amine }\end{array}$} & \\
\hline & 5-HT & $\begin{array}{l}\text { Bufoten- } \\
\text { idine }\end{array}$ & $\begin{array}{c}\text { Candi- } \\
\text { cine }\end{array}$ & $\begin{array}{c}\text { Lepto- } \\
\text { dactyline }\end{array}$ & & & & & & \\
\hline \multicolumn{11}{|l|}{$\begin{array}{l}\text { Leptodactylinae } \\
\text { Leptodactylus }\end{array}$} \\
\hline \multicolumn{11}{|l|}{ "Pachypus Section" } \\
\hline \multicolumn{11}{|l|}{ A-group } \\
\hline $\begin{array}{l}\text { L. p. pentadactylus } \\
\text { (5) Perú, Iquitos, } \\
\text { 1962, } 9 \text { (AC) }\end{array}$ & 140 & - & 45 & 9 & 15 & 一 & - & - & - & 10 \\
\hline $\begin{array}{l}\text { (1) Venezuela, Cu- } \\
\text { maná, } 1964,6(\mathrm{AC})^{1}\end{array}$ & 230 & - & 7 & 13 & 150 & - & - & - & - & 560 \\
\hline $\begin{array}{l}\text { L. p. dengleri } \\
\text { (1) Costa Rica, Li- } \\
\text { món, 1962, } 5 \text { (AC) }\end{array}$ & 55 & 600 & $2-3 ?$ & 13 & 35 & - & - & - & - & $={ }^{3}$ \\
\hline $\begin{array}{l}\text { (19) Costa Rica, Tur- } \\
\text { rialba, } 1964,8 \text { (AC) }\end{array}$ & 20 & 550 & - & 11 & 25 & - & - & - & - & 40 \\
\hline $\begin{array}{l}\text { L. p. labyrinthicus } \\
\text { (7) Argentina, Mi- } \\
\text { siones, } 1961,2 \text { (AC) }\end{array}$ & 220 & - & - & 1.5 & 100 & 110 & 35 & 20 & 90 & 12 \\
\hline $\begin{array}{l}\text { (5) Argentina, Mi- } \\
\text { siones, 1961, } 9 \text { (AC) }\end{array}$ & 1900 & - & - & 12.5 & 740 & 670 & 230 & 120 & 410 & 640 \\
\hline $\begin{array}{l}\text { (2) Argentina, Mi- } \\
\text { siones, } 1963,12 \text { (AC) }\end{array}$ & 1500 & - & - & $={ }^{3}$ & 48 & 215 & 83 & 15 & 75 & 10 \\
\hline $\begin{array}{l}\text { (1) Argentina, Mi- } \\
\text { siones, 1963, } 12 \text { (F) } \\
\text { (MET) }\end{array}$ & 20 & - & - & $={ }^{3}$ & 12 & 80 & 43 & 4 & 32 & $==^{3}$ \\
\hline $\begin{array}{l}\text { (5) Argentina, Mi- } \\
\text { siones, 1966, 9 (F) } \\
\text { (MET) }\end{array}$ & 70 & - & - & $<0.1$ & 36 & 13 & 6.5 & 23 & 26 & 275 \\
\hline $\begin{array}{l}\text { (1) Brazil, Minas Ge- } \\
\text { rais, 1966, } 5 \text { (MET) }\end{array}$ & 1900 & - & - & 8 & 420 & 310 & 90 & 5.5 & - & $={ }^{3}$ \\
\hline $\begin{array}{l}\text { (8) Brazil, Minas Ge- } \\
\text { rais, 1966, } 11 \text { (MET) }\end{array}$ & 1500 & - & - & 30 & 720 & 740 & 80 & 23 & 51 & 775 \\
\hline $\begin{array}{l}\text { (5) Brazil, Minas Ge- } \\
\text { rais, 1966, 11 (F) } \\
\text { (MET) }\end{array}$ & 200 & - & - & 0.35 & 90 & 95 & 5 & 3.5 & 6.5 & 630 \\
\hline $\begin{array}{l}\text { (14) Brazil, Minas } \\
\text { Gerais, 1966, } 12 \text { (F) } \\
\text { (MET) }\end{array}$ & 50 & - & - & 1.3 & 36 & 37 & 6.5 & 5.5 & 3 & 3 \\
\hline $\begin{array}{l}\text { Leptodactylus laticeps } \\
\text { (1) Argentina, For- } \\
\text { mosa, 1960, } 3 \text { (AC) }\end{array}$ & 320 & - & - & $2-3$ & 75 & - & - & $=3$ & - & $={ }^{3}$ \\
\hline $\begin{array}{l}\text { (1) Argentina, For- } \\
\text { mosa, 1961, I (AC) }\end{array}$ & 280 & - & - & $2-3$ & 160 & - & - & $5-10$ & - & $={ }^{3}$ \\
\hline $\begin{array}{l}\text { (1) Argentina, For- } \\
\text { mosa, 1966, 1 (F) } \\
\text { (AC) }\end{array}$ & 340 & - & - & $={ }^{3}$ & 100 & - & - & 4 & - & 850 \\
\hline $\begin{array}{l}\text { (6) Argentina, For- } \\
\text { mosa, 1966, } 3 \text { (MET) }\end{array}$ & 40 & - & - & $={ }^{3}$ & 55 & - & - & 一 & 一 & 110 \\
\hline
\end{tabular}


Table 4. (Continued.)

\begin{tabular}{|c|c|c|c|c|c|c|c|c|c|c|}
\hline \multirow[b]{3}{*}{$\begin{array}{c}\text { Taxa, sample size, locality, } \\
\text { date }\end{array}$} & \multirow{2}{*}{\multicolumn{2}{|c|}{$\begin{array}{c}\text { Indolealkyl- } \\
\text { amines }\end{array}$}} & \multirow{2}{*}{\multicolumn{2}{|c|}{$\begin{array}{l}\text { Phenylalkyl- } \\
\text { amines }\end{array}$}} & \multicolumn{5}{|c|}{ Imidazolealkylamines } & \multirow{3}{*}{$\begin{array}{c}\text { Poly- } \\
\text { peptides } \\
\text { (caeru- } \\
\text { lein- } \\
\text { like) }\end{array}$} \\
\hline & & & & & \multirow[b]{2}{*}{$\begin{array}{c}\text { Hista- } \\
\text { mine }\end{array}$} & \multirow{2}{*}{$\begin{array}{l}\mathrm{N}- \\
\text { Methyl } \\
\text { hista- } \\
\text { mine }\end{array}$} & \multirow{2}{*}{$\begin{array}{l}\text { N,N-Di- } \\
\text { methyl } \\
\text { hista- } \\
\text { mine }\end{array}$} & \multirow{2}{*}{$\begin{array}{c}\text { Spinace- } \\
\text { amine }\end{array}$} & \multirow{2}{*}{$\begin{array}{l}\text { 6- } \\
\text { Methyl- } \\
\text { spinace- } \\
\text { amine }\end{array}$} & \\
\hline & 5-HT & $\begin{array}{c}\text { Bufoten- } \\
\text { idine }\end{array}$ & $\begin{array}{c}\text { Candi- } \\
\text { cine }\end{array}$ & $\begin{array}{c}\text { Lepto- } \\
\text { dactyline }\end{array}$ & & & & & & \\
\hline $\begin{array}{l}\text { (2) Argentina, For- } \\
\text { mosa, 1966, } 4(\mathrm{~F}) \\
(\mathrm{MET})\end{array}$ & 100 & - & - & 0.5 & 150 & - & - & - & - & 620 \\
\hline $\begin{array}{l}\text { (2) Argentina, For- } \\
\text { mosa, 1966, } 5(\mathrm{~F}) \\
(\mathrm{MET})\end{array}$ & 90 & - & - & 0.5 & 100 & - & - & - & - & 670 \\
\hline $\begin{array}{l}\text { (2) Argentina, For- } \\
\text { mosa, 1966, } 6(\mathrm{~F}) \\
(\mathrm{MET})\end{array}$ & 240 & - & - & 0.5 & 240 & - & - & - & - & 1500 \\
\hline B-group & & & & & & & & & & \\
\hline L. bolivianus & & & & & & & & & & \\
\hline $\begin{array}{l}\text { (1) Penú, Iquitos, } \\
1962,11 \text { (AC) }\end{array}$ & - & - & 一 & 480 & - & - & 一 & - & 一 & 一 \\
\hline $\begin{array}{l}\text { (1) Perú, Iquitos, } \\
1965,1 \text { (AC) }\end{array}$ & 一 & - & - & 420 & - & 一 & - & - & - & - \\
\hline $\begin{array}{l}\text { L. ocellatus } \\
\text { a) }\end{array}$ & & & & & & & & & & \\
\hline $\begin{array}{l}\text { (59) Brazil, Guana- } \\
\text { bara, 1962, 1 (AC) }\end{array}$ & 一 & 一 & - & 490 & - & - & 一 & - & 一 & 一 \\
\hline $\begin{array}{l}\text { (1) Brazil, S. Bernardo } \\
\text { do Campo, 1962, } 4 \\
\text { (AC) }\end{array}$ & - & 一 & 一 & 750 & 一 & - & - & - & - & - \\
\hline $\begin{array}{l}\text { (1050) Brazil, S. } \\
\text { Paulo, (4 pools) } \\
\text { 1957-58, } 1 \text { (AC) }\end{array}$ & - & - & $\overline{\text { (rant }}$ & $\begin{array}{l}770-1150 \\
\text { ge of } 4 \text { pools }\end{array}$ & s) & - & - & - & - & - \\
\hline $\begin{array}{l}\text { (5) Argentina, Mi- } \\
\text { siones, Iguazú, 1961, } \\
2 \text { (AC) }\end{array}$ & - & - & 一 & 340 & - & 一 & - & 一 & 一 & 一 \\
\hline $\begin{array}{l}\text { (3) Argentina, Mi- } \\
\text { siones, Oberá, 1961, } \\
2 \text { (AC) }\end{array}$ & - & - & 一 & 615 & - & - & - & - & 一 & 一 \\
\hline $\begin{array}{l}\text { (3) Argentina, Ituza- } \\
\text { ingó, } 1961,2 \text { (AC) }\end{array}$ & - & - & - & 202 & - & - & 一 & - & - & - \\
\hline $\begin{array}{l}\text { (3) Argentina, Corri- } \\
\text { entes, 1961, 2 (AC) }\end{array}$ & - & - & 一 & 530 & - & - & - & - & - & - \\
\hline b) & & & & & & & & & & \\
\hline $\begin{array}{l}\text { (3) Argentina, Chaco, } \\
\text { Resistencia, 1961, } 9 \\
\text { (AC) }\end{array}$ & - & - & - & 2860 & - & - & - & - & - & 一 \\
\hline $\begin{array}{l}\text { (5) Argentina, Chaco, } \\
\text { Resistencia, 1962, } 10 \\
\text { (AC) }\end{array}$ & - & - & 一 & $1540(S)_{1}^{2}$ & - & - & - & - & - & - \\
\hline $\begin{array}{l}\text { (2) Argentina, Chaco, } \\
\text { Resistencia, 1962, } 10 \\
\text { (AC) }\end{array}$ & - & - & - & $8800(S)_{2}^{2}$ & 一 & - & - & - & 一 & 一 \\
\hline
\end{tabular}


Table 4. (Continued.)

\begin{tabular}{|c|c|c|c|c|c|c|c|c|c|c|}
\hline \multirow[b]{3}{*}{$\begin{array}{c}\text { Taxa, sample size, locality, } \\
\text { date }\end{array}$} & \multirow{2}{*}{\multicolumn{2}{|c|}{$\begin{array}{l}\text { Indolealkyl- } \\
\text { amines }\end{array}$}} & \multirow{2}{*}{\multicolumn{2}{|c|}{$\begin{array}{l}\text { Phenylalkyl- } \\
\text { amines }\end{array}$}} & \multicolumn{5}{|c|}{ Imidazolealkylamines } & \multirow{3}{*}{$\begin{array}{c}\text { Poly- } \\
\text { peptide } \\
\text { (caeru- } \\
\text { lein- } \\
\text { like) }\end{array}$} \\
\hline & & & & & & & N,N-Di- & & & \\
\hline & 5-HT & $\begin{array}{l}\text { Bufoten- } \\
\text { idine }\end{array}$ & $\begin{array}{l}\text { Candi- } \\
\text { cine }\end{array}$ & $\begin{array}{l}\text { Lepto- } \\
\text { dactyline }\end{array}$ & $\begin{array}{l}\text { Hista- } \\
\text { mine }\end{array}$ & $\begin{array}{l}\text { hista- } \\
\text { mine }\end{array}$ & $\begin{array}{l}\text { hista- } \\
\text { mine }\end{array}$ & $\begin{array}{l}\text { Spinace- } \\
\text { amine }\end{array}$ & $\begin{array}{l}\text { spinace- } \\
\text { amine }\end{array}$ & \\
\hline
\end{tabular}

(6) Argentina, Chaco, Resistencia, 1962, 11

(AC)

(5) Argentina, Chaco, Resistencia, 1963, 1

(AC)

(2) Argentina, Buenos Aircs, 1962, 6 (AC)

(8) Argentina, La

Plata, 1960, 8 (AC)

(10) Argentina, Bahía Blanca, 1962, 10 (AC)

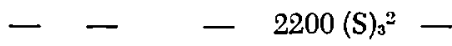

(6) Uruguay, Montevideo, 1962, 11 ( $\mathrm{AC}$ )

(99) Brazil, Porto

Alegre, ( 3 pools)

(AC) $1956-57,1$

(3) Argentina, Córdoba, 1962, 2 ( AC)

(63) Argentina, Mendoza, ( 7 pools ) 196162,1 ( AC)

c)

- $\quad 2860$

$-4400$

- 3080

$-\quad 2500$

$-\quad 2500$

$$
\text { - 2300-2600 - }
$$

(range of 3 pools)

(26) Argentina, San Luis, Mnt. $1000 \mathrm{mtr}$. (4 pools) 1959 ( AC)

L. chaquensis $-\quad 1430$ - 725-2400 ( range of 7 pools)

(25) Argentina, Formosa, ( 2 pools) 1960 , 2 (AC)

(5) Argentina, Jujuy, 1960,3 (AC)

(215) Argentina, Tucumán, ( 10 pools) 1960-62 (AC)

(16) Argentina, Corrientes, 1962, 7 (AC) (23) Argentina, Corrientes, 1960, 2 (AC)

(19) Argentina, Corrientes, Iberá, 1961, 2 ( AC)

(12) Argentina, Corrientes, 1961, 2 (AC)

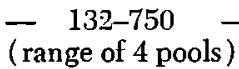

- 50-72

$-\quad 110$

- 87-220

(range of 10 pools) 
Table 4. (Continued.)

\begin{tabular}{|c|c|c|c|c|c|c|c|c|c|}
\hline \multirow[b]{3}{*}{ Taxa, sample size, locality, } & \multirow{3}{*}{$\begin{array}{c}\begin{array}{c}\text { Indolealkyl- } \\
\text { amines }\end{array} \\
\text { 5-HT Bufoten- } \\
\text { idine }\end{array}$} & \multirow{2}{*}{\multicolumn{2}{|c|}{$\begin{array}{l}\text { Phenylalkyl- } \\
\text { amines }\end{array}$}} & \multicolumn{5}{|c|}{ Imidazolealkylamines } & \multirow{3}{*}{$\begin{array}{c}\text { Poly- } \\
\text { peptide } \\
\text { (caeru- } \\
\text { lein- } \\
\text { like) }\end{array}$} \\
\hline & & & & & $\mathrm{N}-$ & N,N-Di- & & 6- & \\
\hline & & $\underset{\text { cine }}{\text { Candi- }}$ & $\begin{array}{l}\text { Lepto- } \\
\text { dactyline }\end{array}$ & $\begin{array}{l}\text { Hista- } \\
\text { mine }\end{array}$ & $\begin{array}{l}\text { hista- } \\
\text { mine }\end{array}$ & $\begin{array}{l}\text { hista- } \\
\text { mine }\end{array}$ & $\begin{array}{l}\text { Spinace- } \\
\text { amine }\end{array}$ & $\begin{array}{l}\text { spinace- } \\
\text { amine }\end{array}$ & \\
\hline
\end{tabular}

(11) Argentina, Chaco, Resistencia, 1961, 10 (AC)

(16) Argentina, Chaco, Resistencia, 1962, 10 (AC)

(2) Argentina, Chaco, Benitez, 1962, 10 (AC)

(42) Argentina, Chaco, Resistencia, 1962, 11

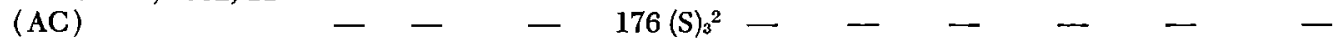

1 (F), fresh skins; (MET), methanol extraction; (AC), acetone extraction. ${ }^{2}(S)_{1},(S)_{2},(S)_{3}$ : sympatric populations. $\mathbf{s}=$, not investigated.

tyrosine to para- and meta-tyramine; $N$ methyltransferase $(s)$, responsible for the transformation of the primary amine into the secondary and tertiary amine, and then into the quaternary ammonium base; $N$ acetylase, transforming histamine into $\mathrm{N}$ acetylhistamine; and finally enzymes responsible for the cyclisation of bufotenine to dehydrobufotenine, possibly via a paraquinonid structure, and of $N$-methylhista- mine, or $N, N$-dimethylhistamine to spinaceamine and 6-methylspinaceamine, respectively.

The question must be left open of whether there exists a single decarboxylase, active on all aromatic aminoacids, or different decarboxylases, with higher substrate specificity. Similar problems may be posed for the $N$-methyltransferases and for the enzymes responsible for cyclisation.

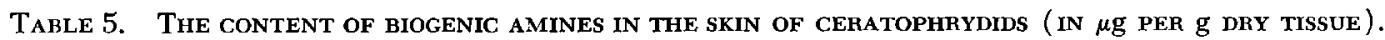

\begin{tabular}{lcc}
\hline \multicolumn{1}{c}{ Taxa, sample size, locality, date } & $\begin{array}{c}\text { Indolealkylamines } \\
\text { (5-1/T) }\end{array}$ & $\begin{array}{c}\text { Phenylalkylamines } \\
\text { (leptodactyline) }\end{array}$ \\
\hline $\begin{array}{l}\text { Ceratophrys ornata } \\
\text { (3) Argentina, Santiago del Estero, 1961, 2 (AC) }\end{array}$ & - & 1.5 \\
(2) Argentina, Tucumán, 1961, 12 (F) (MET) & - & 3.3 \\
$\begin{array}{l}\text { Lepidobatrachus asper } \\
\text { (5) Argentina, Santa Fé, 1963, 12 (MET) }\end{array}$ & 5 & 7 \\
Lepidobatrachus salinicola & & \\
(1) Argentina, Santa Fé, 1963, 12 (MET) & & 11 \\
Lepidobatrachus llanensis & - & 0.5 \\
(3) Argentina, La Rioja, 1964, 2 (MET) & $310-710$ & \\
Odontophrynus americanus & 1700 & 7 \\
(27) Argentina, Tucumán, (2 pools), 1960, 4 (AC) & 270 & 5 \\
(5) Argentina, Tucumán, 1961, 2 (AC) & & \\
(12) Uruguay, 1966, 3 (AC) & 6400 & 1.3 \\
Odontophrynus occidentalis & 5250 & 0.8 \\
(5) Argentina, Mendoza, Malargüe, 1964, 1 (MET) & & \\
(7) Argentina, Mendoza, Malargüe, 1965, 3 (MET) & & \\
\hline
\end{tabular}

' (F), fresh skins; (MET), methanol extraction; (AC), acetone extraction; -, not detected. 


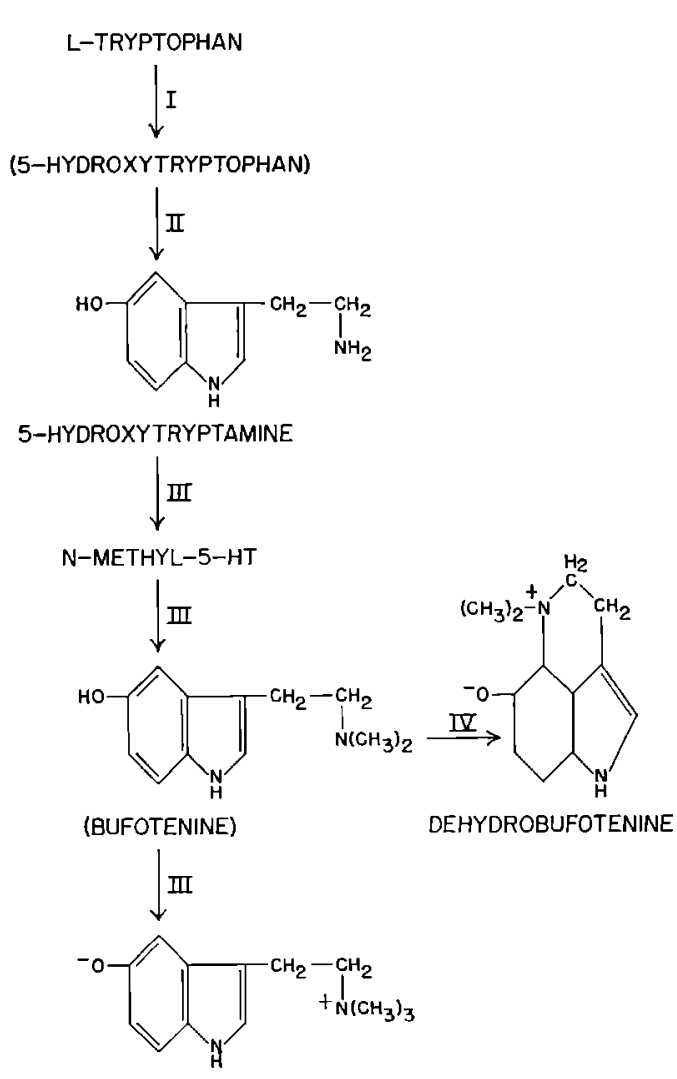

BUFOTENIDINE

FIG. 1.-Biosynthesis of indolealkylamines in the skin of leptodactylid frogs (parentheses indicate compounds as yet not found in the skin); I, tryptophan 5-hydroxylase; II, decarboxylase of aromatic L-amino acids; III, $N$-methyltransferase; IV, enzyme(s) responsible for cyclisation.

The accompanying figures 1 to 3 give a synopsis of the different amines found in the leptodactylid frogs and indicate the enzyme systems involved in their biosynthesis. In parentheses are indicated obligatory intermediates, not yet found in detectable amounts in our skin extracts.

Concerning physalaemin (Anastasi, Erspamer, and Cei, 1964) and caerulein (Anastasi, Erspamer, and Endean, 1967) it is obvious that several enzymes must play a part in their biosynthesis, rigidly directed by the genetic code.

The amino acid sequence of physalaemin (I) is shown below, together with sequence of eledoisin (II), a pharmacologically closely related endecapeptide found in the posterior salivary glands of Eledone, a Mediterranean octopod.

$$
\begin{aligned}
& \text { Pyr-Ala-Asp-Pro-Asp-Lys-Phe- } \\
& \text { Tyr-Gly-Leu-Met. } \mathrm{NH}_{2} \\
& \text { Pyr-Pro-Ser-Lys-Asp-Ala-Phe- } \\
& \text { Ilu-Gly-Leu-Met. } \mathrm{NH}_{2}
\end{aligned}
$$

It may be seen that both the $\mathrm{N}$-terminal amino acid residue and the $\mathrm{C}$-terminal tripeptide are common to the two endecapeptides.

Similarly, the sequence of caerulein (III) is indicated below, together with the sequence of gastrin II (IV), one of the hormonal principles responsible for the secretion of gastric juice.

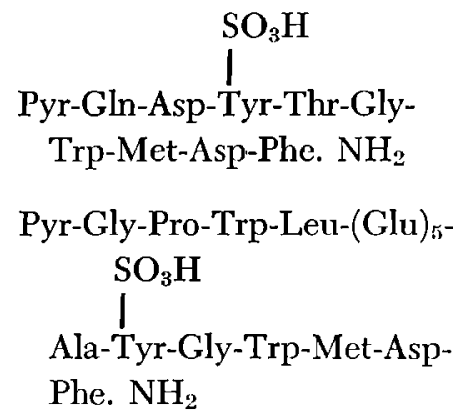

It is astonishing that the $\mathrm{N}$-terminal amino acid residue, the C-terminal pentapeptide, and the $O$-sulphate of the tyrosinyl residue are common to the two polypeptides. Moreover, it should be stressed that the common C-terminal pentapeptide is necessary and sufficient to produce a potent stimulation of the gastric secretion.

For the present it seems prudent to simply report the above objective data, without any premature speculation.

Caerulein is the polypeptide isolated from the skin of the Australian tree-frog $\mathrm{Hyla}$ caerulea. The problem of whether caeruleinlike polypeptides from Leptodactylus are identical or not with caerulein must be left open. Similarly, sequence IV is peculiar for human gastrin-II. Gastrins from other animal species may show substitutions of apparently non-essential amino acid residues. 


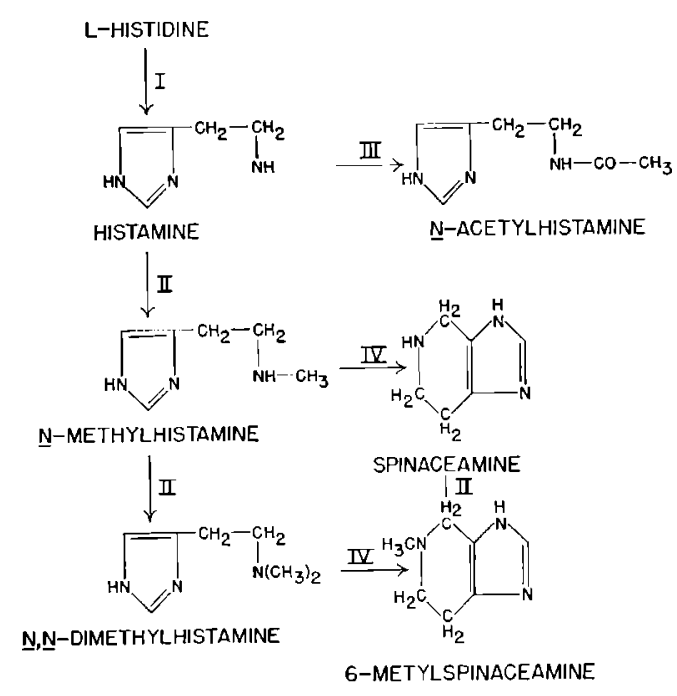

FIG. 2.-Biosynthesis of imidazolealkylamines in the skin of leptodactylid frogs; I, histidin decarboxylase (s); II, $N$-methyltransferase; III, $N$-acetylase; IV, enzyme(s) responsible for cyclisation.

\section{II}

It may be seen from tables 1 and 2 that subfamilies such as Telmatobiinae, Calyptocephalellinae, Elosiinae, Eleutherodactylinae, Cyclorhamphinae, and Batrachylinae, show similarities in their consistently extreme scarcity of aromatic amines and polypeptides (tables 1 and 2). Only in a few cases could a little amount of 5-HT or leptodactyline be detected (cf. Thoropa, Calyptocephalella). A relative taxonomic independence of all these leptodactylid stocks has been pointed out (Gallardo, 1965) and supposedly they were "primitive," as the ancestral stock of bufonid-like frogs (Noble, 1931). Moreover, affinities between the Batrachylinae (Eupsophus, Batrachyla), the Eleutherodactylinae, and the Cyclorhamphinae has been repeatedly reported. However, a special position of Rhinoderma darwini, by virtue of the very high content of 5-HT of its skin, appears evident.

On the other hand, the natural appearance of the subfamily Leptodactylinae, to
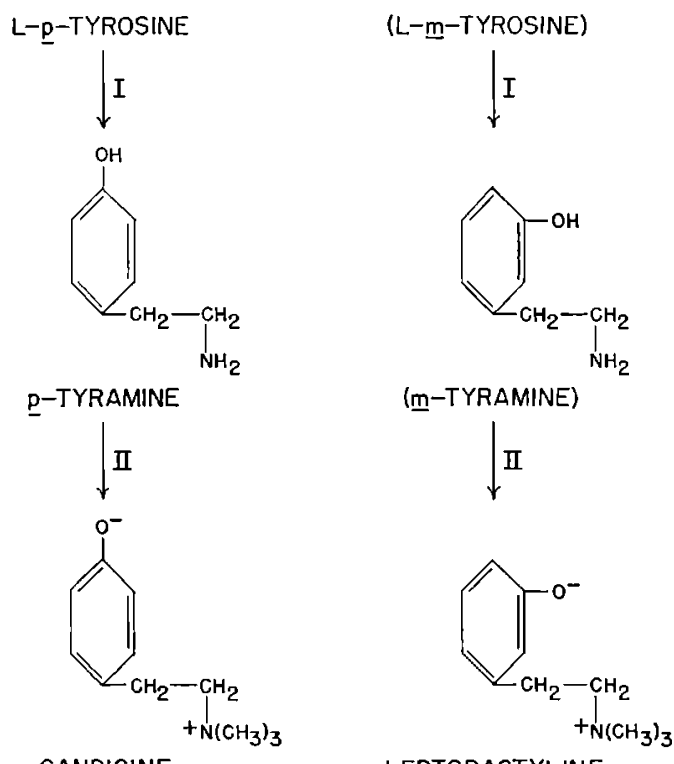

ANDICINE

LEPTODACTYLINE

FIG. 3.-Biosynthesis of phenylalkylamines in the skin of leptodactylid frogs (parentheses indicate compounds as yet not found in the skin); I, decarboxylase of aromatic L-amino acids; II, $N$-methyltransferase.

which the genera Leptodactylus, Physalaemus, Eupemphix, Engystomops, and Pleurodema belong, has been pointed out (Noble, 1931; Gallardo, 1965). Edalhorina, Lythodites, Limnomedusa, and Pseudopaludicola likely may be referred to this group.

Our biochemical data indicate a striking heterogeneity of skin constituents of the Leptodactylinae, comparable only to the great variety of their life histories. Physalaemus, Pleurodema, and Eupemphix ("Paludicola" of former authors, in the broad sense) were considered a possible "ancestor group" by Noble. They do not contain more aromatic amines than the Telmatobiinae or the other subfamilies, excepting the Rhinodermatinae. However, Physalaemus contains a powerful eledoisinlike polypeptide, physalaemin, very active on vascular and extravascular smooth muscle. It is interesting that indolealkylamines, such as 5-HT, practically lacking in the skin of these anurans, may be detected in remarkable amount only in some concen- 
trated glandular structures (inguinal glands of Pleurodema bufonina), by which high tissue specialization during specific morphogenetic differentiation may be inferred.

The most varied spectrum of biogenic amines exists in Leptodactylus species and the parts of this spectrum correspond astonishingly to the bio-morphological sections established by Lutz (1931) for this polymorphic genus. A clear cut differentiation may be seen between the so-called "Cavicola," including a number of terrestrial species burrowing their foam nests underground, and the other sections. The general bio-morphological uniformity of "Cavicola" is supported by our biochemical data indicating a general lack of biogenic amines and polypeptides (Table 3). On the contrary, the "Platymantis" and "Pachypus" sections show a great variety of representatives of all groups of biogenic amines here considered, in addition to the presence, in some stocks, of physiologically active polypeptides of the caerulein type. Relationships between $L$. caliginosus podicipinus, L. c. petersi, and L. melanonotus can be inferred from the evident similarity of their indole- and phenyl-alkylamines content. L. rubido and L. curtus from Peruvian high-Amazonian lands, probably related to the rare giant form L. vilarsi (Cei, 1965: in press ), are suggested by their amine spectrum to be members of the "Platymantis" group. But morphological features (inguinal glands, sex characters) and the presence of polypeptides of the caerulein type suggest a special position as a somewhat independent stock, perhaps intermediate between "Pachypus" and "Platymantis" (Table 3).

The "Pachypus" section presents the most interesting results both from a general biochemical point of view (occurrence of $\mathrm{N}$ methylated histamine and tyramine derivatives) and from the evolutionary point of view. Two morphological groups can be easily distinguished. To the first belong the giant $L$. pentadactylus, probably also $L$. fallax and $L$. flavopictus, and probably, $L$. laticeps. To the second group belong $L$. bolivianus, L. ocellatus, and L. chaquensis (L. macrosternum is likely an allied populational stock of chaquensis).

A glance at Table 4 is sufficient to make clear the striking comparability of morphological date (cf: Gallardo, 1964) with present biochemical data.

The skins of L. pentadactylus pentadactylus, L. p. labyrinthicus, and L. p. dengleri show a fundamentally similar spectrum of biogenic amines, characterized by abundance of indolealkylamines and imidazolealkylamines, with relative scarcity of phenylalkylamines. However, whereas the skin of L. $p$. dengleri can methylate 5-IIT to bufotenidine and that of L. p. pentadactylus and L. p. dengleri can carry out the methylation of $p$-tyramine to candicine, that of L. p. labyrinthicus, and partially also that of L. laticeps, is capable not only of $N$-methylating and $N$-acetylating histamine to $N$-methyl and $N, N$-dimethyl-histamine, and to $N$-acetylhistamine, respectively, but also to produce a linkage of the lateral chain to the imidazole nucleus, giving origin to the two imidazo- $c$-pyridine derivatives spinaceamine and 6-methylspinaceamine.

Our biochemical screening could thus contribute substantially to the recognition of these closely related forms as species rather than subspecies if their geographical relation were better known. In our opinion, the above striking differences in the $N$ methyltransferase activity of the skin of $L$. p. pentadactylus, L. p. labyrinthicus, and $L$. $p$. dengleri, as evidenced by the different substrates acted upon by the enzyme(s), are of such value that a specific status of these frogs could be more easily supported than the relative position of discussed species such as $L$. podicipinus, L. rubido, $L$. curtus, and L. melanonotus.

Tryptophan-5-hydroxylase activity is lacking or very low in all the ocellatus-like frogs, bolivianus, ocellatus, and chaquensis, in which, on the contrary, $N$-methyltransferase is very active. Acting on the excellent substrate represented by $m$-tyramine the enzyme causes the formation of enor- 
mous amounts of the quaternary ammonium base leptodactyline. $N$-methylation seems to be particularly intense in $L$. ocellatus, in which concentrations of leptodactyline as high as $8000 \mu \mathrm{g} / \mathrm{g}$ dry skin may be reached.

From Table 4 it may be seen that the leptodactyline content of the skin of the widespread species L. ocellatus shows some geographic variation (Fig. 4). One populational area is an eastern tropical one from Brazil (Rio de Janeiro) to Argentina (Corrientes), and is characterized by a relatively lower content of the ammonium base (pools range $202-750 \mu \mathrm{g} / \mathrm{g}$ ). A South-central temperate area is alongside the Paraná borders, taking in the $L$. ocellatus populations with the highest content of leptodactyline in the skin (pools range: 725-8800 $\mu \mathrm{g}$ / g). An isolated population in the central mountains of Argentina (San Luis Sierras, 1000-1200 meters) shows the lowest leptodactyline content of the skin $(132-750 \mu \mathrm{g}$ / $\mathrm{g})$. We can suppose a genetic regulation of such biochemical variation, but its ecological significance and its adaptative value are still unknown.

A comparison between the sibling species L. ocellatus and L. chaquensis in their allosympatric range is very interesting. Data from Table 4 show that in the area of sympatry, on the Paraná River, the two species show a striking difference in the leptodactyline content of their skin. In fact, in $L$. ocellatus and $L$. chaquensis specimens collected by us simultaneously, in the same ecological environment, the leptodactyline content of the skin was 1540,8800 , and $2200 \mu \mathrm{g} / \mathrm{g}$, and 220,530 , and $176 \mu \mathrm{g} / \mathrm{g}$, respectively. It should be pointed out that the biological and taxonomic position of the two species under discussion is now well established (Cei, 1950; Gallardo, 1964; Cei and Cohen, 1965; Barrio, 1966).

During our screening, no seasonal differences in the leptodactyline content of the skin were definitely demonstrated. Similarly, available data are not yet sufficient to confirm any significant sex difference, in spite of the fact that the amine content ap- peared somewhat higher in female specimens. At any rate, we must recall that our estimations indicate only the static content of amines at a given moment, and that they do not offer any reliable information on the turnover rate of these amines.

To conclude this review, results of Table 5 concerning some ceratophrid genera should be briefly discussed. Whereas Ceratophrys and Lepidobatrachus are very poor in indolealkylamines, Odontophrynus shows a very high content of 5-HT in its skin. This result is in accord with previous observations on the relative position of the ceratophrid genera, the remarkable distance of Odontophrynus from the other neotropical representatives of this family having been stressed by morphological (Reig, 1959; Gallardo, 1965) and serological arguments (Cei, 1965).

Acknowledgment.-This work was supported by grants from the Consiglio $\mathrm{Na}$ zionale della Ricerche, Rome, Italy.

\section{LITERATURE}

Anastasi, A., V. Erspamer, and G. Bertaccini. 1965. Occurrence of bradykinin in the skin of Rana temporaria. Comp. Biochem. Physiol., 14: 43-52.

Anastasi, A., V. Erspamer, and J. M. Cei. 1964. Isolation and amino acid sequence of physalaemin, the main active polypeptide of the skin of Physalaemus fuscumaculatus. Arch. Biochem. Biophys., 108:341-348.

Anastasi, A., V. Enspamer, and R. Endean. 1967. Isolation and structure of caerulein, an active peptide from the skin of Hyla caerulea. Experientia, 23:699-700.

Barnio, A. 1966. Divergencia acústica entre el canto nupcial de Leptodactylus ocellatus (Linné) y L. chaquensis Cei (Anura, Leptodactylidae). Physis, 26, no. 72:275-277.

Berg, C. 1896. Batracios Argentinos. Anal. Museo Nac. Bs. Aires, V, 2:147-226.

Bertaccini, G, J. M. Cei, and V. Erspamer, 1965a. Occurrence of physalaemin in extracts of the skin of Physalaemus fuscumaculatus and its pharmacological actions on extra-vascular smooth muscle. Brit. J. Pharmacol., 25:363-379.

Bertaccini, G., J. M. CeI, and V. Erspamer. 1965b. The action of physalaemin on the systemic arterial blood pressure of some experimental animals. Brit. J. Pharmacol., 25:380391.

Burger, W. L. 1954. Two family groups of Neotropical frogs. Herpetologica, 10:194-196. 
CEI, J. M. 1965. Observaciones preliminares sobre las formas alto-amazonicas en Leptodactylus del grupo rubido-vilarsi. Acta III. Congr. Sudamericano Zoología, Santiago de Chile, Octubre, 1965, Inv. Zool. Chil. (in press).

CeI, J. M., AND R. Cohen. 1965. Serological relationships in the Leptodactylus (pachypus) species group. Copeia, 2:155-158.

Cei, J. M., and V. Espamer. 1966. Biochemical taxonomy of South American amphibians by means of skin amines and polypeptides. Copeia, 1:74-78.

Erspamer, V., G. Bertaccini, and N. Urakawa. 1964. Biogenic amines and active polypeptides in the skin of ten Japanese amphibian species. Jap. J. Pharmacol., 14:468-473.

Erspamer, V., J. M. Cer, and M. Roseghini. 1963. Occurrence of candicine (p-hydroxyphenylethyltrimethylammonium) in extracts of the skin of Leptodactylus pentadactylus pentadactylus. Life Sciences, No. 11:825-827.

Erspamer, V., G. De Caro, and R. Endean. 1966. Occurrence of a physalaemin-like polypeptide (uperolein) and other active polypeptides in the skin of Uperoleia rugosa. Experientia, 22:738.

Erspamer, V., and G. Falconteri Erspamer. 1962. Pharmacological actions of eledoisin on extravascular smooth muscle. Brit. J. Pharmacol., 19:337-354.

Erspamer, V., and A. GläsSER. 1963. The action of eledoisin on the systemic arterial blood pressure of some experimental animals. Brit. J. Pharmacol., 20:516-527.

Enspamer, V., M. Roseghini, and J. M. Cei. 1964. Indole-, imidazole-, and phenyl-alkylamines in the skin of thirteen Leptodactylus species. Biochem. Pharmacology, 13:1083-1093.

Erspamer, V., M. Roseghini, R. Endean, and A. ANASTASI. 1966. Biogenic amines and active polypeptides in the skin of Australian amphibians. Nature, 212:204.

Eirspamer, V., T. Vitali, M. Roseghini, and J. M. CEI. 1963. Occurrence of new imidazole-alkylamines (spinaceamine and 6-methylspinaceamine) in skin extracts of Leptodactylus pentadactylus labyrinthicus. Experientia, 19:346-347.

Erspamer, V., T. Vitali, M. Roseghini, and J. M.
CEI. 1964. The identification of new histamine derivatives in the skin of Leptodactylus. Arch. Biochem. Biophys., 105:620-629.

Erspamer, V., T. Vitali, M. Roseghini, and J. M. CEr. 1967. 5-Methoxy- and 5-hydroxyindoles in the skin of Bufo alvarius. Biochem. Pharmacol., 16:1149-1164.

Gallardo, J. M. 1964. Consideraciones sobre Leptodactylus ocellatus (L.) (Amphibia, Anura) y especies aliadas. Physis, 24, no. 68:373-384.

Gallardo, J. M. 1965. A propósito de los Leptodactylidae (Amphibia, Anura). Papéis Avulsos Dep. Zoología. S. Paulo, 17, 8:77-87.

GoIN, J. C., AND O. B. GoIv. 1962. Introduction to Herpetology. Freeman and Co., San Francisco. i-vii $+341 \mathrm{pp}$.

LuTz, A. 1931. Segunda memoria sobre especies brasileiras do genero Leptodactylus incluindo outras aliadas. Mem. Inst. O. Cruz, 23:1-20.

LuTz, B. 1954. Anfibios Anuros do Distrito Federal. Mem. Inst. O. Cruz, 52, 1:155-238.

Miranda Ribeiro, A. 1926. Notas para servirem ao estudo dos Gymnobatrachios (Anura) brasileiros. Arch. Mus. Nac. Rio de Janeiro, 27:227.

Noble, G. K. 1931. Biology of the Amphibia. McGraw-Hill, New York. xii +577 pp.

REIG, O. A. 1959. Las relaciones genéricas del Anuro chileno Calyptocephalella gayi. Actas y Trab. $I^{\circ}$ Congr. Sudamer. Zoologia, La Plata, Octubre 1959, IV:113-131.

ReIg, O. A. 1959. La Anatomia esquelética del genero Lepidobatrachus (Anura, Leptodactylidae) comparada con la de otros Ceratofrinos. Actas y Trab. $I^{\circ}$ Congr. Sudamer. Zoologia, La Plata, Octubre 1959, IV:133-147.

Savage, J. M., and A. L. de Carvalho. 1953. The family position of Neotropical frogs currently referred to the genus Pseudis. Zoologica, 38: 193-200.

VELLARD, J. 1951. Estudios sobre batracios Andinos. I. El grupo Telmatobius y formas afines. Mem. Mus. Hist. Nat. J. Prado, 1:1-89.

Institute of Biology, National University of Cuyo, Mendoza, Argentina, and Institute of Pharmacology, University of Parma, Parma, Italy. 